\title{
COMBATing Breast Cancer: Conference on Molecular Basics and Therapeutic Implications in Breast Cancer
}

\author{
Gesprächsleitung: Achim Rody ${ }^{a}$ Dieter Niederacher ${ }^{b}$ \\ Teilnehmer: Gunter von Minckwitz ${ }^{c}$ Christoph Thomssen ${ }^{d} \quad$ Andreas Schneeweiss $^{\mathrm{e}}$ \\ Manfred Schmitt ${ }^{f}$ \\ ${ }^{a}$ Klinik für Gynäkologie und Geburtshilfe, Johan-Wolfgang-Goethe-Universität, Frankfurt/M., \\ ${ }^{\mathrm{b}}$ Frauenklinik, Universitätsklinikum Düsseldorf, \\ ' GBG Forschungs GmbH, Neu-Isenburg, \\ ${ }^{d}$ Klinik und Poliklinik für Gynäkologie, Martin-Luther-Universität Halle-Wittenberg, Halle/Saale, \\ e Universitäts-Frauenklinik, Universitätsklinikum Heidelberg, \\ ${ }^{f}$ Frauenklinik, Klinikum rechts der Isar, Technische Universität München, Germany
}

Frage 1: Welche Fragestellungen und Ziele der Grundlagenforschung und der translationalen Forschung sind in den nächsten Jahren am vordringlichsten, welche erscheinen am erfolgversprechendsten? Welches sind derzeit die molekularbiologischen «Hot Topics»?

von Minckwitz: Die Zahl der Forschungsrichtungen ist sehr groß und es ist schwierig, vorab die erfolgversprechendste zu identifizieren. Wichtige Themen erscheinen mir die Stammzellforschung und die Untersuchung des InsulinstoffwechselPathways.

Thomssen: Die folgenden Fragen halte ich für besonders wichtig: Welches sind die Ursachen von Brustkrebs? Kann die Früherkennung von Brustkrebs mittels eines einfachen Bluttestes erreicht oder verbessert werden? Ist eine weitere Verbesserung der Prognoseabschätzung möglich, um die - gegenwärtig häufige - Übertherapie zu verringern? Ist dies nur durch komplexe Algorithmen unter Einbeziehung von histopathologischen Befunden, klinischen Befunden und molekularen Markern möglich? Ist der Einsatz von multiparametrischen Gen-, Protein- oder Methylierungsprofilen notwenig? Werden in Zukunft auch Einzelmarker noch eine Rolle spielen? Kann es durch den Einsatz von Multiparameterprofilen gelingen, Einzelmarker (auf Gen- oder Proteinebene) herauszufiltern, welche für sich alleine spezifische klinische Fragestellungen beantworten? Welche verbesserten Möglichkeiten der Prädiktion der Wirkung verschiedener Therapien gibt es, um gezielte Therapien zu ermöglichen? Lassen sich neben Proliferation und Apoptose weitere «Pathways» und Signalkaskaden entschlüsseln, welche für die Regulation der Ma- lignität der Tumorzellen eine Rolle spielen? Ist die Umkehr der malignen Transformation und Eigenschaften einer Zelle reversibel und wie könnte dies vermittelt werden?

Schneeweiss: Für den Kliniker ganz im Vordergrund steht die verbesserte Steuerung der Systemtherapie solider Tumoren durch Evaluation und Validierung verbesserter prognostischer und prädiktiver Faktoren, um Unter-, Über- und Fehltherapie zu vermindern. Molekulare Signaturen auf genomischer, epigenomischer und transkriptionaler Ebene erscheinen hier neben der Verfeinerung der klassischen pathologischen, insbesondere der immunhistochemischen Parameter derzeit am erfolgversprechendsten.

Schmitt: Meines Erachtens sind die folgenden Bereiche besonders wichtig: MicroRNAs: Dies sind zirka 23-nt-RNAs welche eine bedeutende genregulierende Rolle bei Menschen, Tieren und Pflanzen spielen. Sie binden an mRNAs von proteincodierenden Genen mit dem Ziel der posttranskriptionellen Repression. Die Aufklärung der Regulation der micro-RNA/ mRNA-Interaktion unter physiologischen und pathophysiologischen Bedingungen (z.B. bei Krebs) ist ein wesentliches Ziel der translationalen Forschung.

Epigenetik: Erforschung des die epigenetischen Veränderungen des Genoms bestimmende Epigenoms, z.B. durch Analyse des Methylierungsstatus der CpG-Inseln in der Promoterregion der DNA. Hierbei werden Methylgruppen an Cytosin-Basen in der Promoterregion eines Gens gekoppelt, in der die Transkription beginnt. Ist die Promoterregion methyliert, ist das Gen in der Regel inaktiv. Epigenetische Mutationen, welche die Methylierung beeinflussen, können für die

\section{KARGER}

Fax +497614520714

Information@Karger.de

www.karger.com (c) 2009 S. Karger GmbH, Freiburg

Accessible online at:

www.karger.com/brc
PD Dr. med. Achim Rody

Klinik für Gynäkologie und Geburtshilfe

Johan-Wolfgang-Goethe-Universität

Theodor-Stern-Kai 7, 60590 Frankfurt/M., Germany

Tel. +49 69 6301-4117, Fax -83469

achim.rody@em.uni-frankfurt.de 
Krebsentstehung eine bedeutende Rolle spielen. Es gibt erste therapeutische Ansätze, die Hypermethylierung der Kontrollgene zu verhindern, indem die für die Übertragung der Methylgruppen zuständigen Enzyme, die DNA-Methyltransferasen, gehemmt werden.

Krebs-Stammzellen: Stammzellen sind pluripotent, sie können zu den verschiedenen Zelltypen ausdifferenzieren, aus denen ihr jeweiliges Gewebe aufgebaut ist. Beim Krebsgeschehen ist bekannt, dass Tumoren aus verschiedenen Zellen bestehen, u.a. einer kleinen Anzahl von unsterblichen Tumorstammzellen, die ständig den Nachschub an Krebszellen liefern. Sie sind relativ resistent gegenüber Chemotherapien und Bestrahlungen. Peripher zirkulierende Tumorzellen: Die Bedeutung von im peripheren Blut zirkulierenden, disseminierten Tumorzellen wird zur Zeit dahingehend untersucht, ob die Zahl der zirkulierenden Tumorzellen und deren Phäno- und Genotyp Auskunft über Therapieansprechen und Verlauf der Krebskrankheit geben können.

Krebs-Biomarker: Ziel ist es, im Blut zirkulierende bzw. im Tumorgewebe verankerte Krebs-Biomarker zu identifizieren und charakterisieren. Solche Biomarker können als Marker für Diagnose, Prognose, Monitoring, Imaging, Therapieansprechen oder Beschreibung von unerwünschten Medikamentennebenwirkungen dienen, aber auch als Zielort der Therapie.

Frage 2: Klinische Studien erproben neue molekulare Substanzen häufig an einem nicht oder unzureichend selektionierten Patientenkollektiv. Welche Mittel und Wege haben wir, dies zu ändern?

von Minckwitz: Am wirksamsten wäre es, wenn Zulassungsbehörden die Identifikation eines Targets zur Prädiktion des Wirksmechanismus mit Nachweis der Reduktion der «Needed Number of Patients to Treat» als gewichtiges Kriterium für eine Zulassung festlegten.

Thomssen: Klinische Studien an unselektierten Patientinnen sind einfach durchzuführen und dadurch in der täglichen Praxis viel leichter zu etablieren. Die Teilnahme an solchen Studien ist im Rahmen einer effektiven Nutzung der Ressourcen, insbesondere unter dem Aspekt «zertifiziertes Brustzentrum», gefragt. Der gezielte Einsatz molekularer Substanzen erfordert eine entsprechende Untersuchung des Tumors, der dafür häufig speziell asserviert und aufbereitet werden muss. Jedes Verfahren erfordert eine besondere Logistik und eine besondere Wachsamkeit der behandelnden Ärzte. Studien mit einem derartig komplexen Design erfordern ein besonderes Engagement und eine besondere Motivation aller Beteiligten und sind in Zeiten der Überlastung mit Dokumentations- und Administrationsaufgaben schwer umzusetzen. Nur durch spezielle Fortbildung und Ausbildung der Kollegen und durch Bereitstellung ausreichender finanzieller und personeller Ressourcen wird auf Dauer eine Verbesserung zu erreichen sein.
Schneeweiss: In intelligent geplanten präklinischen und frühen klinischen Studien müssen neben dem Toxizitätsprofil in ausgefeilten translationalen Begleitprogrammen prädiktive Parameter etabliert werden, die eine Selektion der Patienten erlauben, welche bei Behandlung mit dem neuen Molekül nicht nur einen marginalen Vorteil sondern einen substanziellen Nutzen haben. Bevor viel Geld in große, teure multizentrische Studien investiert wird, muss durch Patientenselektion die Wahrscheinlichkeit eines Nutzens der neuen Substanz drastisch erhöht werden, sonst droht trotz guter Wirksamkeit bei einzelnen Patienten der Wirkungsnachweis im unselektionierten Patientenkollektiv unterzugehen.

\section{Frage 3: Das Wissen um die Wirkungsweise vieler neuer Substanzen ist lückenhaft und vielfach er- weisen sich die vermuteten Targets nicht als die entscheidenden. Könnten «global-molekulare» Unter- suchungen im Rahmen der Patientenversorgung mit einem begleitenden wissenschaftlichen Konzept hilfreich sein?}

von Minckwitz: Ich bezweifele, ob man mit solchen Ansätzen die «Nadel im Heuhaufen» wirklich findet. Die derzeit bahnbrechenden zielgerichteten Substanzen beim Mammakarzinom (Tamoxifen, Trastuzumab) sind anders gefunden worden.

Thomssen: Global-molekulare Untersuchungen im Rahmen begleitender wissenschaftlicher Projekte von klinischen Studien können durchaus hilfreich sein, um Hypothesen über neue Targets zu entwickeln und neue «Zielmoleküle» zu entdecken. Auf jeden Fall ist immer die Entwicklung eines Einzeltestes für das jeweilige Zielmolekül und eine prospektive Validierung notwendig.

Schneeweiss: Die Natur ist viel komplexer, als wir uns vorstellen können. Wir dürfen uns niemals nur mit der Messung eines Parameters oder Targets begnügen, sondern müssen immer ganze Systeme betrachten, um der Biologie einer Tumorzelle näherzukommen. Selbst Trastuzumab wirkt trotz HER2-Überexpression nur bei zirka jeder dritten Frau. Molekulare Signaturen könnten hier einen Ausweg darstellen, erfordern aber eine konsequent gemäß gültigen Standards durchgeführte Evaluation und Validierung. Bevor die Ergebnisse aus abschließenden prospektiven Studien vorliegen, sollten molekulare Signaturen im klinischen Alltag nicht als Entscheidungshilfen benutzt werden.

Schmitt: Sicherlich können global-molekulare Untersuchungen hilfreich sein, vorerst allerdings auf der Genebene, und nicht auf der Proteinebene. Auf Genebene (DNA-Mutationen, mRNA, micro-RNA, DNA-Methylierung) stehen Array-TestKits zur Analyse des menschlichen Genoms zur Verfügung, auf der Proteinebene ist dies nur unvollkommen möglich. Hier 
bestehen vereinzelt Test-Kits, die meistens auf Antikörpern, Inhibitoren, Rezeptoren oder Liganden basieren.

\section{Frage 4: Wie ist der Stellenwert der Pharmaindustrie in der translationalen Forschung zu beurteilen, wie könnten «Investigator-initiated-Trials» (IIT) verstärkt gefördert werden und sind sie finanzierbar?}

von Minckwitz: Zur Pharmaindustrie: siehe mein Kommentar zur Zulassung (Frage 2). Zu den IIT: Es wäre z.B. sinnvoll, Laborforschungsprojekte nur noch dann öffentlich $\mathrm{zu}$ fördern, wenn sie im engen Zusammenhang mit einer klinischen Studie stehen.

Thomssen: Investigator-initiierte Studien können grundsätzlich durch die Industrie finanziert und gefördert werden. Allerdings ist zu bedenken, dass der finanzielle Rahmen ausreichend weit gesteckt werden muss. $\mathrm{Zu}$ bedenken ist weiterhin, dass der zeitliche Rahmen z.B. wegen des Auslaufens der Patente der interessierenden Medikamente häufig beschränkt ist. Unbedingt ist zu fordern, dass öffentliche Förderungsinstitutionen wie die Deutsche Forschungsgemeinschaft (DFG), das Bundesministerium für Bildung und Forschung, die Deutsche Krebshilfe und andere den Zugang zu Forschungsmitteln im Bereich Mammakarzinom erleichtern.

Schneeweiss: Ohne Unterstützung der forschenden Pharmaindustrie wird es keinen substanziellen Fortschritt in der translationalen Forschung geben. Wir sind Partner, gerade jetzt, wo auch die pharmazeutische Industrie aufgrund der Vielzahl neuer Moleküle in der präklinischen und frühen klinischen Entwicklung einem immer stärkeren Konkurrenzkampf ausgesetzt ist. Sie muss dringend nach Prädiktoren für ihr jeweiliges Produkt suchen, um im Markt bestehen zu können. Ich glaube, der Wille der pharmazeutischen Industrie im Rahmen dieser Partnerschaft auch IIT zu fördern wird stetig größer, je mehr Erfolge die Grundlagenforschung bei der Entwicklung neuer «Werkzeuge» vorweisen kann. Angesichts der zum Teil enormen Kostenersparnis in der weiteren Entwicklung und der Gewinnaussichten bei der späteren Vermarktung ist die Evaluation und Validierung neuer Prädiktoren nicht nur finanzierbar, sondern wird eine Grundvoraussetzung für neue Medikamentenentwicklung schlechthin sein.

Schmitt: Durch Etablierung von wissenschaftlichen Netzwerken zwischen Industrie und einzelnen Kliniken, bzw. Forschungsinstituten können IIT gefördert werden. Große, globale Netzwerke sind oft nicht erfolgreich, da die Infrastruktur viel Ressourcen auffrisst. Das Interesse der Industrie an der Zusammenarbeit mit akademischen Institutionen ist immer noch gegeben, wird aber durch die Änderung des Patentrechtes vor einigen Jahren zunehmend erschwert.
Frage 5: Welche strukturellen Änderungen sind zur Stärkung des Wissenschaftsstandortes Deutschland in Bezug auf das Mammakarzinom am vordringlichsten?

von Minckwitz: Mehr Zentralisierung und eine bessere Ausstattung der Kliniken für klinische Studien, wie z.B. in England sind die wichtigsten Maßnahmen.

Thomssen: Die ärztlichen Aufgaben müssen entschlackt und von dem derzeitigen Übermaß an Administrations- und Dokumentationsaufgaben befreit werden. Wir Ärzte müssen wieder den Kopf frei bekommen, um über die medizinischen Probleme unserer Patienten differenziert und kreativ nachzudenken. Im medizinischen wie im naturwissenschaftlichen Bereich sollte bereits im Studium auf die Bedeutung translationaler Forschung hingewiesen werden. In den forschenden Kliniken sollten Stellen für ein bis zwei Naturwissenschaftler speziell für die translationale Forschung vorgesehen werden.

Schneeweiss: Es fehlt an der Möglichkeit, rasch und unbürokratisch eine Förderung für ein vielversprechendes aber eben noch nicht etabliertes Projekt zu erhalten. Dies gilt zum Teil für private Drittmittelgelder, viel stärker aber für öffentliche Fördergelder. Der Beantragungsprozess für die Initialförderung muss vereinfacht werden, gerne auch gekoppelt mit einer dann konsequent und kompromisslos erfolgenden Begutachtung der Ergebnisse nach der ersten Förderperiode.

Schmitt: Gewebe-/Datenbanken müssen etabliert werden und der Austausch von Geweben und Daten ermöglicht bzw. vereinfacht werden. Dies und auch das Follow-up sollte durch die Universitäten finanziert werden. Leider ist dies nur an wenigen Universitäten der Fall.

\section{Frage 6: Wie sind Situation und Perspektiven des wis- senschaftlich-klinischen Nachwuchses zu beurteilen und wie können Verbesserungen erzielt werden?}

von Minckwitz: Hier sehe ich ein besonderes Problem, das sich aus dem Nachwuchsproblem in der Medizin überhaupt ergibt, sich dann aber noch vergrößert, weil an den Universitätskliniken eigentlich kein Freiraum mehr für Forschung durch Ärzte existiert. Gleichzeitig verlangen Arbeitszeitgesetz etc., dass diese Forschung während der Arbeitszeit erfolgen muss. Dies passt einfach nicht zusammen.

Thomssen: Wissenschaftliche Medizin erscheint in Studium und Beruf nicht mehr attraktiv. Weder in der täglichen Arbeit noch als Berufsziel wird die Beschäftigung mit klinischer Forschung als förderlich für die persönlichen Interessen angesehen. Medizinstudium und Arbeitsbedingungen müssen darauf eingerichtet werden, die Motivation für den klinischen 
Nachwuchs zu verbessern. Solange der Arzt allerdings zum Schichtarbeiter degradiert bleibt, ist dies nicht zu erreichen.

Schneeweiss: Die Situation ist gerade für den translational orientierten, klinischen Nachwuchs schwierig, da die Doppelbelastung Klinik und Forschung billigend in Kauf genommen wird. Hier müssen strukturelle Veränderungen erfolgen, die es dem Nachwuchs ähnlich dem US-amerikanischen Modell stärker erlauben, klinische Ausbildung und translationale Forschung zu verbinden, z.B. durch sequentielle allein auf Klinik oder Forschung ausgerichtete Ausbildungsabschnitte innerhalb gut organisierter, kooperativer Gruppen.

Schmitt: Die Perspektiven sind schlecht, da kaum Rotationsstellen für Kliniker in die Forschung zur Verfügung stehen. Zudem ist der forschende Kliniker in der Gynäkologie bei seinen Kollegen nicht besonders geschätzt. Die DFG/Krebshilfe sollte da spezielle Mittel zur Verfügung stellen. Leider ist die akademische Karriere an Universitätskliniken nach der Professorenbesoldungsreform nicht mehr erstrebenswert. Es fehlt einfach unter diesen schlechten Bedingungen der motivierte Nachwuchs.

Frage 7: Der Fortschritt im Hinblick auf das Krankheitsverständnis und die daraus resultierenden Therapieansätze erfordern eine engere Zusammenarbeit von Grundlagenforschern, Pathologen und Klinikern. Wie kann diese gestärkt werden und wo sind die größten Schwächen?

von Minckwitz: Wir versuchen Grundlagenforscher und Pathologen möglichst früh in die Planung unserer klinischen Studien einzubinden. Dann können deren Ideen rechtzeitig Eingang in das Protokoll finden und die Identifikation mit dem Projekt wird verbessert. Dies ist meines Erachtens die Richtung, in die wir noch intensiver gehen müssen.

Thomssen: Auf nationaler und internationaler Ebene (deutsche Studiengruppen, EORTC usw.) gibt es Ansätze, klinische Studienfragestellungen mit translationalen Fragestellungen zu verbinden. Problematisch ist dabei, dass klinische Studiengruppen und translationale Forschungsgruppen häufig nebeneinander arbeiten und die Expertise der jeweiligen Gegenseite nicht genutzt wird. Die Kommunikation, das gegenseitige Verständnis und die Verbesserung dieser Kooperationsansätze müssen gefördert werden. Der gegenseitige Gewinn aus einer solchen Kooperation muss herausgestellt werden.

Schneeweiss: Die Zusammenarbeit kann am besten gestärkt werden durch kooperative Gruppen, in denen jeder Partner gleichberechtigt gehört wird und sich nach außen repräsentiert fühlt. Eine der größten Schwächen im heutigen System ist die mangelnde Transparenz der Leistungen («Erfolg hat viele Väter, Misserfolg nur einen»).

Schmitt: Durch gemeinsame Forschungsprojekte und Rotationsstellen für den motivierten Kliniker (siehe Frage 6) und durch gemeinsame wissenschaftliche Publikationen und Vorträge auf internationalen Konferenzen kann die Kooperation gefördert werden. Studienbegleitende Aktivitäten sind keine translationale Forschung, aber da gibt es bei jungen Kollegen oft ein Missverständnis.

\section{Frage 8: Welche politischen und strukturellen Ver- änderungen sind notwendig, um die Verbindung von Grundlagenforschung und Klinik zu stärken?}

von Minckwitz: Professionelle Strukturen, so wie wir sie z.B. in der German Breast Group (GBG) aufgebaut haben, sind meines Erachtens notwendig, um den heutigen Ansprüchen klinischer und translationaler Forschung gerecht zu werden. Diese sollten von der Politik anerkannt und unterstützt werden.

Schneeweiss: Die Etablierung von Comprehensive Cancer Centers nach US-amerikanischem Vorbild ist ein erster entscheidender Schritt. Diese Idee in Deutschland in ausgesuchten Hochburgen umzusetzen gegen den Widerstand erstarrter Strukturen und veralteter politischer Denkmuster ist Grundvoraussetzung, um im internationalen Wettbewerb auf Dauer bestehen zu können.

Schmitt: Dringend notwendig sind die Stärkung der äußeren Bewertung der wissenschaftlichen Arbeit und die Fortbildung junger Kliniker durch ärztliche Leitung, Dekanat, Klinikum und Kollegen. In vielen Bereichen werden innerhalb der Kliniken wissenschaftlich tätige Kollegen von ihren in der Ausbildung höherstehenden Kollegen unterdrückt und benachteiligt. Zudem kostet wissenschaftliche Arbeit Zeit, die der klinischen Ausbildung zumindest im Frühstadium der Ausbildung verloren geht.

\section{Frage 9: Wie könnte die Verbindung von molekularer/ klinischer Forschung und Therapie optimiert werden?}

von Minckwitz: Siehe meine Antwort zu Frage 8.

Schneeweiss: Siehe meine Antwort zu Frage 8.

Thomssen: Die Kliniker müssen mehr von der Notwendigkeit translationaler Forschung überzeugt werden. Dies bedeutet auch, dass im Medizinstudium wieder mehr Betonung auf die naturwissenschaftliche Ausbildung gelegt werden sollte. Umgekehrt müssen die Naturwissenschaftler noch mehr motiviert 
werden, sich mit den klinischen Fragestellungen vertraut zu machen. Auch dies könnte im jeweiligen Studiengang mit entsprechenden Kursen vorbereitet werden.

Schmitt: Durch Einbeziehung von in der translationalen Grundlagenforschung tätigen Wissenschaftlern/Klinikern bei der Erstellung und Bewertung von klinischen Protokollen, auch bei der Bewertung des potentiellen Einsatzes von Testmethoden und der zu untersuchenden Biomarker kann die Zusammenarbeit optimiert werden. Ebenso bei der Planung von Aufbau und Pflege von Biobanken.

Frage 10: Viele wissenschaftliche Forschungsgruppen arbeiten auf verschiedenen Teilgebieten mehr oder weniger isoliert. Wie wichtig ist die Bildung zentrumsübergreifender Strukturen für eine erfolgreiche translationale Forschung? Wie kann sie erreicht werden?

von Minckwitz: Ich würde eher die Bildung themenbezogener kooperierender Netzwerke, gegebenenfalls mit Zentralstrukturen, befürworten. Das Verständnis zwischen den Teilgebieten ist meist nicht vorhanden und auch nicht erzwingbar. Aber es muss nicht zu einem Thema an mehreren Stellen völlig unabhängig von einander geforscht werden. Das ist Ressourcenvergeudung.

Thomssen: An erster Stelle steht der Wille zur Kooperation. Sinnvolle Strukturen, wie die Erstellung virtueller Tumorund Datenbanken, sind ein erster Schritt, werden aber alleine nicht weiterführen. Ein Mehrwert aus Forschungsergebnissen würde sich auch ergeben, wenn erreicht werden kann, die Forschungsergebnisse bei einzelnen Tumorentitäten auf andere Erkrankungen zu übertragen. Dies kann nur in enger Kooperation mit anderen Fachgebieten gelingen.
Schneeweiss: Die Bildung zentrumsübergreifender Strukturen ist enorm wichtig. Dies sollte durch den Aufbau kooperativer, überregionaler, transparenter Gruppen erfolgen, in die der engagierte, vielversprechende Nachwuchs frühzeitig integriert wird, bevor er in veraltete Denkmuster verfällt.

Schmitt: Solche Strukturen sind sehr wichtig. Durch gemeinsame Forschungsprojekte, Tagungen, Symposien, Personalaustausch, Fortbildungsveranstaltungen vor Ort können sie gefördert werden. Natürlich kostet dies Geld und Zeit.

\section{Teilnehmer}

Prof. Dr. med. Gunter von Minckwitz

GBG Forschungs $\mathrm{GmbH}$

Schleussnerstrasse 42

63263 Neu-Isenburg, Germany

Tel. +49 6102 7480-411, Fax -111

Gunter.vonMinckwitz@germanbreastgroup.de

Prof. Dr. med. Christoph Thomssen

Klinik und Poliklinik für Gynäkologie

Martin-Luther-Universität Halle-Wittenberg

Ernst-Grube-Strasse 40

06097 Halle / Saale, Germany

Tel. +49 345 557-1847, Fax -1501

christoph.thomssen@medizin.uni-halle.de

Prof. Dr. med. Andreas Schneeweiß

Universitätsklinikum Heidelberg

Universitäts-Frauenklinik

Voßstr. 9

69115 Heidelberg, Germany

Tel. +49 $62215635-552$

andreas_schneeweiss@med.uni-heidelberg.de

Univ.-Prof. Dr. rer. nat. Manfred Schmitt

Klinikum rechts der Isar, Technische Universität München,

Ismaninger Strasse 22

81675, München, Germany

Tel. +49 89 4140-2427, Fax -7410

manfred.schmitt@lrz.tum.de 